\title{
Restricted Generalized Nash Equilibria and Controlled Penalty Algorithm ${ }^{1}$
}

\author{
Masao Fukushima²
}

July 18, 2008; revised November 22, 2008

\begin{abstract}
The generalized Nash equilibrium problem (GNEP) is a generalization of the standard Nash equilibrium problem (NEP), in which each player's strategy set may depend on the rival players' strategies. The GNEP has recently drawn much attention because of its capability of modeling a number of interesting conflict situations in, for example, an electricity market and an international pollution control. However, a GNEP usually has multiple or even infinitely many solutions, and it is not a trivial matter to choose a meaningful solution from those equilibria. The purpose of this paper is two-fold. First we present an incremental penalty method for the broad class of GNEPs and show that it can find a GNE under suitable conditions. Next, we formally define the restricted GNE for the GNEPs with shared constraints and propose a controlled penalty method, which includes the incremental penalty method as a subprocedure, to compute a restricted GNE. Numerical examples are provided to illustrate the proposed approach.
\end{abstract}

Key words. Generalized Nash equilibrium, shared constraints, shadow price, penalty method, restricted GNE.

\section{Introduction}

The generalized Nash equilibrium problem (GNEP) is a generalization of the standard Nash equilibrium problem (NEP), in which each player's strategy set may depend on

\footnotetext{
${ }^{1}$ This work was partially supported by a Grant-in-Aid for Scientific Research from Japan Society for the Promotion of Science.

${ }^{2}$ Department of Applied Mathematics and Physics, Graduate School of Informatics, Kyoto University, Kyoto 606-8501, Japan. E-mail: fuku@i.kyoto-u.ac.jp
} 
the rivals' strategies. The early study of such games dates back at least to Debreu [6] and Arrow and Debreu [1], where a GNEP was called a social equilibrium (problem) or an abstract economy. Rosen [29] considered a special class of GNEPs where all players share common constraints. Harker [15] studied GNEPs via a variational inequality (VI) or a quasi-variational inequality (QVI) reformulation. More recently, Pang and Fukushima [25] proposed some GNEP formulations of multi-leader-follower games.

GNEPs have been used to model various problems in applications. Robinson [27, 28] analyzed the so-called measure of effectiveness in a combat model by formulating the model as a GNEP. Wei and Smeers [30] considered a GNEP constructed from a spatial oligopolistic electricity model and proposed a variational inequality approach to determine a solution of the model. Hobbs and Pang [18] treated oligopolistic electricity models with joint constraints by means of linear complementarity formulations. Contreras, Klusch and Krawczyk [5] also solved electrical market games by way of GNEP formulations. Breton, Zaccour and Zahaf [4] analyzed the joint implementation mechanism of environmental projects by formulating the model as a GNEP. Kesselman, Leonardi and Bonafici [19] dealt with an internet switching problem where users behave selfishly (see [8] for a more rigorous formulation of the model). Pang, Scutari, Facchinei and Wang [26] formulated a power allocation problem in parallel interference channels as a GNEP.

We should remark here that the GNE has often been criticized by the economists as a plausible solution concept of a meaningful economic game, because a GNE is not selfenforceable unlike a Nash equilibrium. Nevertheless the GNEP has steadily expanded the area of applications as mentioned above. In particular, a fairly large portion of the recent study on GNEPs focuses on engineering applications where the aim is to design a well balanced system from a game-theoretic viewpoint $[19,26]$. Moreover, in some economic applications, the GNEP model has been used to design Pigovian taxes, by means of which the regulatory authority may induce the agents to satisfy the common constraints under a non-cooperative setting (see, e.g., [16] and the last paragraph of Section 6 in this paper).

Numerical methods for GNEPs have been developed with different objectives and problem settings. It is well known that a GNEP can be represented as a QVI [15, 25]. By using a gap function for QVIs [13], a GNEP can be further reformulated as an optimization problem whose minimum value is zero. We may apply a global optimization method to solve the latter problem. Instead of a gap function, we may use 
the so-called Nikaido-Isoda function [24] to reformulate a GNEP into an optimization problem with zero minimum value. Another approach is to transform a GNEP into a sequence of NEPs by penalizing those constraints which depend on the other players' strategies, and then solve the resulting NEPs through a VI reformulation [25, 12].

A GNEP usually has multiple or even infinitely many solutions. The abovementioned approaches will find an arbitrary GNE among them. A GNEP with shared, or coupled, constraints is a special but important class of GNEPs. This class of GNEPs was studied by Rosen [29], in which the solution concept called a normalized equilibrium was defined. A normalized equilibrium can be computed by solving a certain VI problem $[8,30]$ or a certain optimization problem involving a Nikaido-Isoda-type function $[17,22]$. Parametrized VI approaches are developed to systematically generate as many GNEs as possible [23]. For more details about numerical methods for GNEPs, see $[10,21]$.

As mentioned above, a GNEP usually has many solutions and the uniqueness of a GNE is expected only under very restrictive assumptions. If there are many GNEs, it will not be enough to find just an arbitrary GNE, since other GNEs may also provide reasonable outcomes of the game. In such a case, we could try to find all GNEs or as many GNEs as possible [23]. An alternative approach is to single out a GNE that has some special property. The normalized equilibrium is such a GNE that the Lagrange multipliers (shadow prices) associated with the shared constraints are equal among all players up to constant factors, and its uniqueness is guaranteed under appropriate conditions [29].

Although such a requirement on the shadow prices may be reasonable in some situations, we may also consider the general case where the relative values of different resources for one player are different from those for another player; for example, we may require that the ratio of shadow prices associated with the common resources is neither too large nor too small for every pair of players. To this end, we will introduce a new solution concept called a restricted GNE. The restricted GNE can naturally be regarded as an extension of Rosen's normalized equilibrium [29], and in fact the latter can be characterized as a special case of the former. Although a restricted GNE may not be unique in general, we may expect that it affords useful insights into outcomes of the GNEP, since it is endowed with more specific features than arbitrary GNEs. Moreover the restricted GNE model may provide a more flexible design tool than the normalized equilibrium model in a subtle conflict situation. 
The purpose of this paper is two-fold. First we present an incremental penalty method for the broad class of GNEPs and show that it can find a GNE under suitable conditions. Next, we formally define the restricted GNE for the GNEPs with shared constraints and propose a controlled penalty method, which includes the incremental penalty method as a subprocedure, to compute a restricted GNE. Numerical examples are provided to illustrate the proposed approach.

Notations: The nonnegative and positive orthants in $\Re^{n}$ are denoted by $\Re_{+}^{n}:=\{x \in$ $\left.\Re^{n} \mid x \geq 0\right\}$ and $\Re_{++}^{n}:=\left\{x \in \Re^{n} \mid x>0\right\}$, respectively. All vectors are column vectors. However, a vector formed by concatenating several vectors, say $x, y, \ldots, z$, is often denoted as $(x, y, \ldots, z)$ instead of $\left(x^{T}, y^{T}, \ldots, z^{T}\right)^{T}$, where $T$ denotes transposition.

\section{Generalized Nash Equilibrium}

We consider a non-cooperative game with $N$ players, and call the $\nu$ th player simply player $\nu$. Let $x^{\nu}$ denote an $n_{\nu}$-vector representing a strategy of player $\nu$, where $n_{\nu}$ is a positive integer. All players' strategies are collectively denoted by a tuple $x=$ $\left(x^{1}, \ldots, x^{N}\right) \in \Re^{n}$, where $n=n_{1}+\cdots+n_{N}$. When we focus on the strategy of a particular player, say $\nu$, among $N$ players, we often write $x=\left(x^{\nu}, x^{-\nu}\right)$, where $x^{-\nu}$ denotes the $\left(n-n_{\nu}\right)$-vector $\left(x^{1}, \ldots, x^{\nu-1}, x^{\nu+1}, \ldots, x^{N}\right)$ that represents the strategies of all players except $\nu$. We will also write $n_{-\nu}=n-n_{\nu}$.

Throughout the paper, we will consider the GNEP where each player $\nu$ solves the following optimization problem with the other players' strategies $x^{-\nu}$ being regarded as exogenous:

$$
\begin{aligned}
& P_{\nu}\left(x^{-\nu}\right): \text { minimize } f^{\nu}\left(x^{\nu}, x^{-\nu}\right) \\
& \text { subject to } g_{i}^{\nu}\left(x^{\nu}, x^{-\nu}\right) \leq 0, \quad i=1, \ldots, m_{\nu} \text {, } \\
& h_{j}^{\nu}\left(x^{\nu}\right) \leq 0, \quad j=1, \ldots, l_{\nu},
\end{aligned}
$$

where $f^{\nu}: \Re^{n} \rightarrow \Re, g_{i}^{\nu}: \Re^{n} \rightarrow \Re, i=1, \ldots, m_{\nu}, h_{j}^{\nu}: \Re^{n_{\nu}} \rightarrow \Re, j=1, \ldots, l_{\nu}$ are continuously differentiable functions such that $f^{\nu}\left(\cdot, x^{-\nu}\right)$ and $g_{i}^{\nu}\left(\cdot, x^{-\nu}\right), i=1, \ldots, m_{\nu}$ are convex for each fixed $x^{-\nu} \in \Re^{n_{-\nu}}$, and $h_{j}^{\nu}, j=1, \ldots, l_{\nu}$ are convex. Thus, problems $P_{\nu}\left(x^{-\nu}\right)$ are convex programming problems for all $\nu=1, \ldots, N$.

For $\nu=1, \ldots, N$, let $g^{\nu}: \Re^{n} \rightarrow \Re^{m_{\nu}}$ and $h^{\nu}: \Re^{n_{\nu}} \rightarrow \Re^{l_{\nu}}$ denote the vector-valued functions whose components are $g_{i}^{\nu}, i=1, \ldots, m_{\nu}$ and $h_{j}^{\nu}, j=1, \ldots, l_{\nu}$, respectively. 
Moreover, let $X^{\nu} \subseteq \Re^{n_{\nu}}$ and $X \subseteq \Re^{n}$ be defined by

$$
X^{\nu}:=\left\{x^{\nu} \in \Re^{n_{\nu}} \mid h^{\nu}\left(x^{\nu}\right) \leq 0\right\}, \quad X:=\prod_{\nu=1}^{N} X^{\nu} .
$$

Then the player $\nu$ 's problem can simply be written as

$$
\begin{aligned}
& P_{\nu}\left(x^{-\nu}\right) \text { : minimize } f^{\nu}\left(x^{\nu}, x^{-\nu}\right) \\
& \text { subject to } g^{\nu}\left(x^{\nu}, x^{-\nu}\right) \leq 0, \\
& x^{\nu} \in X^{\nu} \text {. }
\end{aligned}
$$

The GNEP is to find a tuple $x^{*}=\left(x^{*, 1}, \ldots, x^{*, N}\right)$ such that $x^{*, \nu}$ solves problem $P_{\nu}\left(x^{*,-\nu}\right)$ for each $\nu=1, \ldots, N$. Any such tuple is called a generalized Nash equilibrium or GNE for short.

Notice that the constraints of $P_{\nu}\left(x^{*,-\nu}\right)$ depend on the other players' strategies $x^{-\nu}$. The constraints $g_{\nu}\left(x^{\nu}, x^{-\nu}\right) \leq 0$ will particularly be referred to as the dependent constraints. If the dependent constraints do not exist in any player's problem, then the GNEP reduces to the classical NEP, and a GNE is nothing but a Nash equilibrium $(\mathrm{NE})$.

\section{Assumptions and KKT Systems}

For the system of inequalities defining the constraints of problems $P_{\nu}\left(x^{-\nu}\right), \nu=$ $1, \ldots, N$, we say that the extended Mangasarian-Fromovitz constraint qualification (e-MFCQ) holds at $x=\left(x^{1}, \ldots, x^{N}\right) \in X$ if there exists a vector $z=\left(z^{1}, \ldots, z^{N}\right) \in \Re^{n}$ such that

$$
\begin{aligned}
\nabla_{x^{\nu}} g_{i}^{\nu}\left(x^{\nu}, x^{-\nu}\right)^{T} z^{\nu}<0, & \forall i \in \mathcal{I}_{\nu}\left(x^{\nu}, x^{-\nu}\right), \quad \nu=1, \ldots, N, \\
\nabla h_{j}^{\nu}\left(x^{\nu}\right)^{T} z^{\nu}<0, & \forall j \in \mathcal{J}_{\nu}\left(x^{\nu}\right), \quad \nu=1, \ldots, N,
\end{aligned}
$$

where the index sets $\mathcal{I}_{\nu}\left(x^{\nu}, x^{-\nu}\right)$ and $\mathcal{J}_{\nu}\left(x^{\nu}\right)$ are defined by

$$
\begin{gathered}
\mathcal{I}_{\nu}\left(x^{\nu}, x^{-\nu}\right):=\left\{i \mid g_{i}^{\nu}\left(x^{\nu}, x^{-\nu}\right) \geq 0\right\} \subseteq\left\{1, \ldots, m_{\nu}\right\}, \\
\mathcal{J}_{\nu}\left(x^{\nu}\right):=\left\{j \mid h_{j}^{\nu}\left(x^{\nu}\right)=0\right\} \subseteq\left\{1, \ldots, l_{\nu}\right\}
\end{gathered}
$$

for each $\nu=1, \ldots, N$. By a theorem of the alternative, the e-MFCQ can equivalently be stated as follows: For $\nu=1, \ldots, N$, if

$$
\sum_{i \in \mathcal{I}_{\nu}\left(x^{\nu}, x^{-\nu}\right)} \lambda_{i}^{\nu} \nabla_{x^{\nu}} g_{i}^{\nu}\left(x^{\nu}, x^{-\nu}\right)+\sum_{j \in \mathcal{J}_{\nu}\left(x^{\nu}\right)} \mu_{j}^{\nu} \nabla h_{j}^{\nu}\left(x^{\nu}\right)=0
$$


holds for some $\lambda_{i}^{\nu} \geq 0, i \in \mathcal{I}_{\nu}\left(x^{\nu}, x^{-\nu}\right)$, and $\mu_{j}^{\nu} \geq 0, j \in \mathcal{J}_{\nu}\left(x^{\nu}\right)$, then we must have

$$
\begin{gathered}
\lambda_{i}^{\nu}=0, \quad i \in \mathcal{I}_{\nu}\left(x^{\nu}, x^{-\nu}\right), \\
\mu_{j}^{\nu}=0, \quad j \in \mathcal{J}_{\nu}\left(x^{\nu}\right) .
\end{gathered}
$$

As the name suggests, the e-MFCQ is an extension of the Mangasarian-Fromovitz constraint qualification (MFCQ), which is one of the most popular constraint qualifications in constrained optimization [3]. The MFCQ is usually assumed to hold at an optimal solution in order to ensure the existence of Lagrange multipliers satisfying the Karush-Kuhn-Tucker (KKT) conditions. The MFCQ is regarded as a weak condition that normally holds at any feasible solution except for pathological cases. Notice that the e-MFCQ is concerned with a point $x$ that satisfies the individual constraints $h^{\nu}\left(x^{\nu}\right) \leq 0, \nu=1, \ldots, N$, but not necessarily the dependent constraints $g^{\nu}\left(x^{\nu}, x^{-\nu}\right) \leq 0, \nu=1, \ldots, N$. Like the MFCQ, the e-MFCQ may also be considered a mild requirement for the constraints of the optimization problems $P_{\nu}\left(x^{-\nu}\right)$. The eMFCQ will play a crucial role in establishing the finite convergence property of the algorithm presented in the next section (see Theorem 4.1).

Throughout we make the following assumptions on the constraints of the GNEP:

(A1) For each $\nu=1, \ldots, N, X^{\nu}$ is nonempty and compact.

(A2) The e-MFCQ holds at any $x \in X$.

For $\nu=1, \ldots, N$, the KKT conditions for problem $P_{\nu}\left(x^{-\nu}\right)$ can be written as

$$
\begin{aligned}
\mathrm{KKT}_{\nu}: & \nabla_{x^{\nu}} f^{\nu}\left(x^{\nu}, x^{-\nu}\right)+\sum_{i=1}^{m_{\nu}} \lambda_{i}^{\nu} \nabla_{x^{\nu}} g_{i}^{\nu}\left(x^{\nu}, x^{-\nu}\right)+\sum_{j=1}^{l_{\nu}} \mu_{j}^{\nu} \nabla h_{j}^{\nu}\left(x^{\nu}\right)=0, \\
& \lambda_{i}^{\nu} \geq 0, \quad g_{i}^{\nu}\left(x^{\nu}, x^{-\nu}\right) \leq 0, \quad \lambda_{i}^{\nu} g_{i}^{\nu}\left(x^{\nu}, x^{-\nu}\right)=0, \quad i=1, \ldots, m_{\nu}, \\
& \mu_{j}^{\nu} \geq 0, \quad h_{j}^{\nu}\left(x^{\nu}\right) \leq 0, \quad \mu_{j}^{\nu} h_{j}^{\nu}\left(x^{\nu}\right)=0, \quad j=1, \ldots, l_{\nu},
\end{aligned}
$$

where $\lambda_{i}^{\nu}$ and $\mu_{j}^{\nu}$ are Lagrange multipliers. It will be convenient to denote the vectors of Lagrange multipliers as $\lambda^{\nu}=\left(\lambda_{1}^{\nu}, \ldots, \lambda_{m_{\nu}}^{\nu}\right)$ and $\mu^{\nu}=\left(\mu_{1}^{\nu}, \ldots, \mu_{l_{\nu}}^{\nu}\right)$ for each $\nu=$ $1, \ldots, N$.

Since $P_{\nu}\left(x^{-\nu}\right)$ is a convex programming problem, $\mathrm{KKT}_{\nu}$ is a necessary and sufficient optimality condition for $P_{\nu}\left(x^{-\nu}\right)$ under a suitable constraint qualification (including the e-MFCQ). Therefore, a tuple $x=\left(x^{1}, \ldots, x^{N}\right)$ is a GNE if and only if it satisfies the system formed by concatenating $\mathrm{KKT}_{\nu}$ for all $\nu=1, \ldots, N$, which we call the KKT system associated with the GNEP. 


\section{Penalized Reformulation as NEP}

For each $\nu=1, \ldots, N$, let us consider the following problem in which the dependent constraints in $P_{\nu}\left(x^{-\nu}\right)$ are penalized with positive penalty parameters $\rho^{\nu}=$ $\left(\rho_{1}^{\nu}, \ldots, \rho_{m_{\nu}}^{\nu}\right)^{T}$ :

$$
\begin{aligned}
Q_{\nu}\left(x^{-\nu} ; \rho^{\nu}\right): & \operatorname{minimize} \\
& f^{\nu}\left(x^{\nu}, x^{-\nu}\right)+\sum_{i=1}^{m_{\nu}} \rho_{i}^{\nu} g_{i}^{\nu}\left(x^{\nu}, x^{-\nu}\right)_{+} \\
& \text {subject to } \quad h_{j}^{\nu}\left(x^{\nu}\right) \leq 0, \quad j=1, \ldots, l_{\nu},
\end{aligned}
$$

where $g_{i}^{\nu}\left(x^{\nu}, x^{-\nu}\right)_{+}:=\max \left\{0, g_{i}^{\nu}\left(x^{\nu}, x^{-\nu}\right)\right\}$. This is an adaptation of an exact penalty technique in nonlinear programming $[7,14]$ for the GNEP under consideration. Note, however, that violation of the constraints is penalized by using their respective parameters $\rho_{i}^{\nu}$, unlike the conventional penalty technique where the aggregate constraint violation is penalized by using a single penalty parameter. This somewhat exaggerated usage of penalty parameters will play an essential role in designing the controlled penalty method for finding a restricted GNE later in this paper.

The game in which each player $\nu$ solves $Q_{\nu}\left(x^{-\nu} ; \rho^{\nu}\right)$ is a NEP, since the constraints in $Q_{\nu}\left(x^{-\nu} ; \rho^{\nu}\right)$ do not involve the other players' strategies. We denote $\rho=\left(\rho^{1}, \ldots, \rho^{N}\right)$ and refer to this game as $\operatorname{NEP}(\rho)$.

Note that $Q_{\nu}\left(x^{-\nu} ; \rho^{\nu}\right)$ is a convex programming problem for each $\nu$. Specifically, the objective function is continuous in $\left(x^{\nu}, x^{-\nu}\right)$ and convex with respect to $x^{\nu}$ for each fixed $x^{-\nu}$, and the feasible set $x^{\nu}$ is nonempty and compact by Assumption (A1). Hence $\operatorname{NEP}(\rho)$ has an equilibrium for any $\rho$, see e.g., Theorem 1 in $[2, \S 9.1]$.

By introducing artificial variables $\xi_{i}^{\nu}, i=1, \ldots, m_{\nu}, Q_{\nu}\left(x^{-\nu} ; \rho^{\nu}\right)$ can be rewritten as

$$
\begin{aligned}
Q_{\nu}\left(x^{-\nu} ; \rho^{\nu}\right): \quad \text { minimize } & f^{\nu}\left(x^{\nu}, x^{-\nu}\right)+\sum_{i=1}^{m_{\nu}} \rho_{i}^{\nu} \xi_{i}^{\nu} \\
\text { subject to } & \xi_{i}^{\nu} \geq g_{i}^{\nu}\left(x^{\nu}, x^{-\nu}\right), \quad i=1, \ldots, m_{\nu} \\
& \xi_{i}^{\nu} \geq 0, \quad i=1, \ldots, m_{\nu} \\
& h_{j}^{\nu}\left(x^{\nu}\right) \leq 0, \quad j=1, \ldots, l_{\nu} .
\end{aligned}
$$


The KKT conditions for problem $Q_{\nu}\left(x^{-\nu} ; \rho^{\nu}\right)$ may be written as follows:

$$
\begin{aligned}
& \nabla_{x^{\nu}} f^{\nu}\left(x^{\nu}, x^{-\nu}\right)+\sum_{i=1}^{m_{\nu}} \lambda_{i}^{\nu} \nabla_{x^{\nu}} g_{i}^{\nu}\left(x^{\nu}, x^{-\nu}\right)+\sum_{j=1}^{l_{\nu}} \mu_{j}^{\nu} \nabla h_{j}^{\nu}\left(x^{\nu}\right)=0, \\
& \rho_{i}^{\nu}-\lambda_{i}^{\nu}-\beta_{i}^{\nu}=0, \quad i=1, \ldots, m_{\nu}, \\
& \xi_{i}^{\nu} \geq g_{i}^{\nu}\left(x^{\nu}, x^{-\nu}\right), \quad \lambda_{i}^{\nu} \geq 0, \quad \lambda_{i}^{\nu}\left(\xi_{i}^{\nu}-g_{i}^{\nu}\left(x^{\nu}, x^{-\nu}\right)\right)=0, \quad i=1, \ldots, m_{\nu}, \\
& \xi_{i}^{\nu} \geq 0, \quad \beta_{i}^{\nu} \geq 0, \quad \beta_{i}^{\nu} \xi_{i}^{\nu}=0, \quad i=1, \ldots, m_{\nu}, \\
& h_{j}^{\nu}\left(x^{\nu}\right) \leq 0, \quad \mu_{j}^{\nu} \geq 0, \quad \mu_{j}^{\nu} h_{j}^{\nu}\left(x^{\nu}\right)=0, \quad j=1, \ldots, l_{\nu},
\end{aligned}
$$

where $\lambda^{\nu}=\left(\lambda_{i}^{\nu}\right), \beta^{\nu}=\left(\beta_{i}^{\nu}\right), \mu^{\nu}=\left(\mu_{i}^{\nu}\right)$ are Lagrange multipliers. By eliminating $\beta^{\nu}$, we may rewrite (1) as

$$
\begin{aligned}
& \nabla_{x^{\nu}} f^{\nu}\left(x^{\nu}, x^{-\nu}\right)+\sum_{i=1}^{m_{\nu}} \lambda_{i}^{\nu} \nabla_{x^{\nu}} g_{i}^{\nu}\left(x^{\nu}, x^{-\nu}\right)+\sum_{j=1}^{l_{\nu}} \mu_{j}^{\nu} \nabla h_{j}^{\nu}\left(x^{\nu}\right)=0, \\
& 0 \leq \lambda_{i}^{\nu} \leq \rho_{i}^{\nu}, \quad i=1, \ldots, m_{\nu}, \\
& \xi_{i}^{\nu} \geq g_{i}^{\nu}\left(x^{\nu}, x^{-\nu}\right), \quad \xi_{i}^{\nu} \geq 0, \quad i=1, \ldots, m_{\nu}, \\
& \lambda_{i}^{\nu}>0 \Rightarrow \xi_{i}^{\nu}=g_{i}^{\nu}\left(x^{\nu}, x^{-\nu}\right), \quad i=1, \ldots, m_{\nu}, \\
& \lambda_{i}^{\nu}<\rho_{i}^{\nu} \Rightarrow \xi_{i}^{\nu}=0, \quad i=1, \ldots, m_{\nu}, \\
& h_{j}^{\nu}\left(x^{\nu}\right) \leq 0, \quad \mu_{j}^{\nu} \geq 0, \quad \mu_{j}^{\nu} h_{j}^{\nu}\left(x^{\nu}\right)=0, \quad j=1, \ldots, l_{\nu} .
\end{aligned}
$$

Notice that we must have $\xi_{i}^{\nu}=\max \left\{0, g_{i}^{\nu}\left(x^{\nu}, x^{-\nu}\right)\right\}$ for all $i=1, \ldots, m_{\nu}$ at an optimal solution of problem $Q_{\nu}\left(x^{-\nu} ; \rho^{\nu}\right)$. This fact implies

$$
\xi_{i}^{\nu}=0 \quad \Rightarrow \quad g_{i}^{\nu}\left(x^{\nu}, x^{-\nu}\right) \leq 0
$$

and

$$
\xi_{i}^{\nu}>0 \quad \Rightarrow \quad g_{i}^{\nu}\left(x^{\nu}, x^{-\nu}\right)>0 .
$$

Therefore, if $\xi_{i}^{\nu}=0$ for all $i$, then by (3), the solution $x^{\nu}$ of the penalty problem $Q_{\nu}\left(x^{-\nu} ; \rho^{\nu}\right)$ is feasible to problem $P_{\nu}\left(x^{-\nu}\right)$, and hence optimal to $P_{\nu}\left(x^{-\nu}\right)$. However, if $\xi_{i}^{\nu}>0$ for some $i$, then by (4), the solution $x^{\nu}$ of the penalty problem $Q_{\nu}\left(x^{-\nu} ; \rho^{\nu}\right)$ is infeasible to problem $P_{\nu}\left(x^{-\nu}\right)$. Moreover, if $\xi_{i}^{\nu}>0$, then $\lambda_{i}^{\nu}=\rho_{i}^{\nu}$ holds by the complementarity condition in (1). From these observations, we may naturally expect that the feasibility is restored by increasing those penalty parameters $\rho_{i}^{\nu}$ which correspond to the constraints such that $\xi_{i}^{\nu}=g_{i}^{\nu}\left(x^{\nu}, x^{-\nu}\right)>0$. In fact, if $\rho_{i}^{\nu}$ is sufficiently large and $\rho_{i}^{\nu}>\lambda_{i}^{\nu}$ holds at an optimal solution of $Q_{\nu}\left(x^{-\nu} ; \rho^{\nu}\right)$, then we have $\xi_{i}^{\nu}=0$ by (2) and hence the constraint $g_{i}^{\nu}\left(x^{\nu}, x^{-\nu}\right) \leq 0$ in $P_{\nu}\left(x^{-\nu}\right)$ will be satisfied.

We state an algorithm for finding a GNE by solving a sequence of NEPs. 


\section{Incremental Penalty Algorithm}

Step 0. Choose initial penalty parameters $\rho_{i}^{0, \nu}>0$ and small positive constants $\Delta_{i}^{\nu}$ for $i=1, \ldots, m_{\nu}, \nu=1, \ldots, N$. Set $k:=0$.

Step 1. Find a NE $x^{k}=\left(x^{k, 1}, \ldots, x^{k, N}\right)$ of $\operatorname{NEP}\left(\rho^{k}\right)$ with $\rho^{k}=\left(\rho_{i}^{k, \nu}\right)$. Let $\lambda^{k}=\left(\lambda_{i}^{k, \nu}\right)$, $\mu^{k}=\left(\mu_{j}^{k, \nu}\right)$ and $\xi^{k}=\left(\xi_{i}^{k, \nu}\right)$ be vectors of Lagrange multipliers and artificial variables that along with $x^{k}$ satisfy the KKT conditions $(2)$ for $\nu=1, \ldots, N$.

Step 2. If $\xi_{i}^{k, \nu}=0$ for all $i=1, \ldots, m_{\nu}$ and $\nu=1, \ldots, N$, then terminate.

Step 3. For each $i=1, \ldots, m_{\nu}, \nu=1, \ldots, N$, let

$$
\rho_{i}^{k+1, \nu}:= \begin{cases}\rho_{i}^{k, \nu}+\Delta_{i}^{k, \nu} & \text { if } \xi_{i}^{k, \nu}>0, \\ \rho_{i}^{k, \nu} & \text { if } \xi_{i}^{k, \nu}=0,\end{cases}
$$

where $\Delta_{i}^{k, \nu}$ are chosen so that $\Delta_{i}^{k, \nu} \geq \Delta_{i}^{\nu}$. Go to Step 1 .

We give a basic convergence result for the Incremental Penalty Algorithm.

Theorem 4.1. Suppose that Assumptions (A1) and (A2) are satisfied. Then the Incremental Penalty Algorithm terminates after a finite number of iterations and produces a solution of the GNEP.

Proof. If the algorithm terminates at the $k$ th iteration, then we have $\xi_{i}^{k, \nu}=0$, and hence $g_{i}^{\nu}\left(x^{k, \nu}, x^{k,-\nu}\right) \leq 0$ for $i=1, \ldots, m_{\nu}, \nu=1, \ldots, N$. This implies that $x^{k, \nu}$ solves not only $Q_{\nu}\left(x^{k,-\nu} ; \rho^{k, \nu}\right)$ but also $P_{\nu}\left(x^{k,-\nu}\right)$ for every $\nu=1, \ldots, N$. Consequently, $x^{k, 1}, \ldots, x^{k, N}$ comprise a GNE. Now suppose that the algorithm does not terminate finitely. Let us define the index sets $J^{k}=\left\{(\nu, i) \mid \xi_{i}^{k, \nu}>0, i=1, \ldots, m_{\nu}, \nu=\right.$ $1, \ldots, N\}$. Then $J^{k} \neq \emptyset$ for all $k$ and there exists an infinite subsequence $\left\{J^{k}\right\}_{k \in K}$ such that $J^{k}$ are identical for all $k \in K$. Let $J^{k}=J^{*}$ for all $k \in K$. Then by the update rule of penalty parameters, we have $\rho_{i}^{k, \nu} \rightarrow \infty$ for each $(\nu, i) \in J^{*}$. Since $\lambda_{i}^{k, \nu}=\rho_{i}^{k, \nu}$ for $(\nu, i) \in J^{k}$, this implies that the sequence of Lagrange multipliers $\left\{\left(\lambda^{k}, \mu^{k}\right)\right\}$ is unbounded. Denote $r^{k}=\left\|\left(\lambda^{k}, \mu^{k}\right)\right\|$. Then we have $r^{k} \rightarrow \infty$, at least on some subsequence. Since the sequence $\left\{x^{k}\right\}$ is bounded by Assumption (A1) and the sequence $\left\{\left(\lambda^{k}, \mu^{k}\right) / r^{k}\right\}$ is contained in the unit sphere and hence is also bounded, dividing both sides of the first equality in (2) by $r^{k}$ and taking the limit of an appropriate subsequence yield

$$
\sum_{i=1}^{m_{\nu}} \hat{\lambda}_{i}^{\nu} \nabla_{x^{\nu}} g_{i}^{\nu}\left(\hat{x}^{\nu}, \hat{x}^{-\nu}\right)+\sum_{j=1}^{l_{\nu}} \hat{\mu}_{j}^{\nu} \nabla h_{j}^{\nu}\left(\hat{x}^{\nu}\right)=0
$$


for all $\nu=1, \ldots, N$, where $\hat{x}$ and $(\hat{\lambda}, \hat{\mu})$ are accumulation points of the sequences $\left\{x^{k}\right\}$ and $\left\{\left(\lambda^{k}, \mu^{k}\right) / r^{k}\right\}$, respectively. Note that $\|(\hat{\lambda}, \hat{\mu})\|=1$ and hence $(\hat{\lambda}, \hat{\mu}) \neq(0,0)$. Moreover, it is not difficult to see that $\hat{\lambda}_{i}^{\nu} \geq 0$ for any $(\nu, i)$ such that $g_{i}^{\nu}\left(\hat{x}^{\nu}, \hat{x}^{-\nu}\right) \geq 0$ and $\hat{\mu}_{j}^{\nu} \geq 0$ for any $(\nu, j)$ such that $h_{j}^{\nu}\left(\hat{x}^{\nu}\right)=0$, while $\hat{\lambda}_{i}^{\nu}=0$ for any $(\nu, i)$ such that $g_{i}^{\nu}\left(\hat{x}^{\nu}, \hat{x}^{-\nu}\right)<0$ and $\hat{\mu}_{j}^{\nu}=0$ for any $(\nu, j)$ such that $h_{j}^{\nu}\left(\hat{x}^{\nu}\right)<0$. Then it follows from Assumption (A2) that $\hat{\lambda}_{i}^{\nu}$ and $\hat{\mu}_{j}^{\nu}$ are all zero. This is a contradiction, and hence the algorithm must terminate finitely.

\section{$5 \quad$ Restricted GNEs}

Theorem 4.1 ensures that the Incremental Penalty Algorithm finds a GNE. However the GNEP generally has multiple or often infinitely many equilibria. It would therefore be useful to extract those GNEs from the whole set of GNEs which are of significance in some sense, and try to find such a GNE rather than an arbitrary GNE. In this section, we introduce a new concept of GNE in which some additional conditions are imposed on the Lagrange multipliers for the dependent constraints.

In the remainder of the paper, we restrict ourselves to the special subclass of GNEPs where the dependent constraints in each player's problem originate from some common constraints. Specifically, we assume that player $\nu$ 's problem is given by

$$
\begin{array}{ll}
\bar{P}_{\nu}\left(x^{-\nu}\right): & \text { minimize } \\
& f^{\nu}\left(x^{\nu}, x^{-\nu}\right) \\
\text { subject to } & g_{i}\left(x^{\nu}, x^{-\nu}\right) \leq 0, \quad i=1, \ldots, m, \\
& h_{j}^{\nu}\left(x^{\nu}\right) \leq 0, \quad j=1, \ldots, l_{\nu},
\end{array}
$$

where $g_{i}: \Re^{n} \rightarrow \Re, i=1 \ldots, m$ are continuously differentiable and $g_{i}\left(\cdot, x^{-\nu}\right): \Re^{n_{\nu}} \rightarrow$ $\Re, i=1, \ldots, m$ are convex for each fixed $x^{-\nu} \in \Re^{n_{-\nu}}$, and $f^{\nu}$ and $h_{j}^{\nu}, j=1, \ldots, l_{\nu}$ are same as before. The difference between $\bar{P}_{\nu}\left(x^{-\nu}\right)$ and $P_{\nu}\left(x^{-\nu}\right)$ is that the dependent constraint functions $g_{i}, i=1, \ldots, m$ in $\bar{P}_{\nu}\left(x^{-\nu}\right)$ are common to all players' problems. The GNEP of this type, which was explicitly introduced by Rosen [29], will be referred to as the GNEP with shared constraints. The GNEP with shared constraints plays an important role in modeling some interesting problems such as electricity market, communication, internet switching and environmental problems $[4,5,19,26,30]$, and has recently been studied by a number of authors $[8,9,17,20,22]$. 
The KKT conditions for problems $\bar{P}_{\nu}\left(x^{-\nu}\right), \nu=1, \ldots, N$ can be written as

$$
\begin{aligned}
\overline{\mathrm{KKT}}_{\nu}: & \nabla_{x^{\nu}} f^{\nu}\left(x^{\nu}, x^{-\nu}\right)+\sum_{i=1}^{m} \lambda_{i}^{\nu} \nabla_{x^{\nu}} g_{i}\left(x^{\nu}, x^{-\nu}\right)+\sum_{j=1}^{l_{\nu}} \mu_{j}^{\nu} \nabla h_{j}^{\nu}\left(x^{\nu}\right)=0, \\
& \lambda_{i}^{\nu} \geq 0, \quad g_{i}\left(x^{\nu}, x^{-\nu}\right) \leq 0, \quad \lambda_{i}^{\nu} g_{i}\left(x^{\nu}, x^{-\nu}\right)=0, \quad i=1, \ldots, m, \\
& \mu_{j}^{\nu} \geq 0, \quad h_{j}^{\nu}\left(x^{\nu}\right) \leq 0, \quad \mu_{j}^{\nu} h_{j}^{\nu}\left(x^{\nu}\right)=0, \quad j=1, \ldots, l_{\nu} .
\end{aligned}
$$

Similarly to the general GNEP, $\overline{\mathrm{KKT}}_{\nu}$ is a necessary and sufficient optimality condition for $\bar{P}_{\nu}\left(x^{-\nu}\right)$ under a suitable constraint qualification, and hence a tuple $x=\left(x^{1}, \ldots, x^{N}\right)$ is a GNE if and only if it along with some Lagrange multipliers $\lambda=\left(\lambda_{i}^{\nu}\right) \in \Re^{m N}$ and $\mu=\left(\mu_{j}^{\nu}\right) \in \Re^{l_{1}+\cdots+l_{N}}$ satisfies the system formed by concatenating $\overline{\mathrm{KKT}}_{\nu}, \nu=1, \ldots, N$.

Note that when the $i$ th shared constraint $g_{i}(x) \leq 0$ is inactive at a GNE, the complementarity condition in $\overline{\mathrm{KKT}}_{\nu}$ implies that the corresponding Lagrange multiplier is zero in each player's problem, i.e., $\lambda_{i}^{\nu}=0$ for all $\nu=1, \ldots, N$.

As mentioned earlier, there are in general (often infinitely) many GNEs in the game. This may be regarded as a kind of weakness of the GNEP model, since it does not provide clear explanation of possible outcomes of the model. Therefore we may try to give a characterization of particular GNEs that are supposed to have some distinctive features. We will do this by imposing a certain additional condition on the Lagrange multipliers $\lambda=\left(\lambda_{i}^{\nu}\right) \in \Re^{m N}$ associated with the shared constraints. Specifically we introduce the new solution concept of the restricted generalized Nash equilibrium.

Let $\Lambda$ be a nonempty cone in $\Re_{+}^{m N}$. A tuple $x=\left(x^{1}, \ldots, x^{N}\right)$ is said to be a $\Lambda$ restricted GNE, or simply a restricted GNE, if there exist Lagrange multipliers $\lambda=$ $\left(\lambda_{i}^{\nu}\right) \in \Re^{m N}$ and $\mu=\left(\mu_{j}^{\nu}\right) \in \Re^{l_{1}+\cdots+l_{N}}$ that satisfy $\overline{\mathrm{KKT}}_{\nu}, \nu=1, \ldots, N$, together with the additional condition

$$
\lambda=\left(\lambda_{i}^{\nu}\right) \in \Lambda \text {. }
$$

Obviously, any restricted GNE is a GNE, but the converse is in general not true.

The class of restricted GNEs contains as a special case the normalized equilibrium introduced by Rosen [29]. Recall that $x=\left(x^{\nu}\right)$ is called a normalized equilibrium if the Lagrange multipliers $\lambda=\left(\lambda_{i}^{\nu}\right) \in \Re^{m N}$ satisfy, in addition to $\overline{\mathrm{KKT}}_{\nu}, \nu=1, \ldots, N$, the condition

$$
\lambda_{i}=\lambda_{i}^{0} \beta, \quad i=1, \ldots, m,
$$

for some $\beta=\left(\beta^{\nu}\right) \in \Re_{++}^{N}$ and $\lambda_{i}^{0} \in \Re_{+}, i=1, \ldots, m$, where $\lambda_{i}=\left(\lambda_{i}^{1}, \ldots, \lambda_{i}^{N}\right) \in \Re^{N}$, 
$i=1, \ldots, m$. Let us consider the closed convex cone $\Lambda_{\beta}^{m}$ defined by

$$
\Lambda_{\beta}^{m}=\prod_{i=1}^{m}\left\{\lambda_{i}^{0} \beta \in \Re_{+}^{N} \mid \lambda_{i}^{0} \geq 0\right\} \subseteq \Re^{m N},
$$

which is also written as

$$
\Lambda_{\beta}^{m}=\overbrace{\Lambda_{\beta} \times \cdots \times \Lambda_{\beta}}^{m},
$$

where $\Lambda_{\beta}=\{t \beta \mid t \geq 0\}$ is the half line in $\Re^{N}$ emanating from the origin and passing through the point $\beta \in \Re_{++}^{N}$. Then we can easily see that a $\Lambda_{\beta}^{m}$-restricted GNE is nothing but a normalized equilibrium. It is shown in [29] that a $\Lambda_{\beta}^{m}$-restricted GNE exists for every given $\beta \in \Re_{++}^{N}$, as long as the game has a nonempty feasible set $\left\{x \in X \mid g_{i}(x) \leq 0, i=1, \ldots, m\right\}$. (Note that the set $X$ is nonempty and compact by Assumption (A1).) Moreover, if the mapping $F_{\beta}: \Re^{n} \rightarrow \Re^{n}$ defined by

$$
F_{\beta}(x)=\left(\begin{array}{c}
\frac{1}{\beta^{1}} \nabla_{x^{1}} f^{1}\left(x^{1}, x^{-1}\right) \\
\vdots \\
\frac{1}{\beta^{N}} \nabla_{x^{N}} f^{N}\left(x^{N}, x^{-N}\right)
\end{array}\right)
$$

is strictly monotone, then the $\Lambda_{\beta}^{m}$-restricted GNE is unique [29].

Note that (5) can be rewritten as

$$
\lambda_{i}^{\nu}=\beta^{\nu} \lambda_{i}^{0}, \quad i=1 \ldots, m, \nu=1, \ldots, N
$$

that is,

$$
\lambda^{\nu}=\beta^{\nu} \lambda^{0}, \quad \nu=1, \ldots, N
$$

where $\lambda^{\nu}=\left(\lambda_{1}^{\nu}, \ldots, \lambda_{m}^{\nu}\right) \in \Re^{m}$ and $\lambda^{0}=\left(\lambda_{1}^{0}, \ldots, \lambda_{m}^{0}\right) \in \Re^{m}$. The relation (6) implies that the Lagrange multiplier vectors $\lambda^{\nu} \in \Re^{m}$ associated with the shared constraints are equal among all players $\nu=1, \ldots, N$, up to constant factors $\beta^{\nu}$. In economic terms, this means that the relative values of shadow prices associated with the common resources are identical for all players at any normalized equilibrium. Such a requirement may be appropriate in some situations, but it may often be too strong, because the relative values of different resources for one player may be different from those for another player. The cone $\Lambda$ used to define the $\Lambda$-restricted GNE may represent various conditions that we require a GNE to meet. For example, we may require that the ratio of shadow prices associated with the common resources is neither too large nor too 
small for every pair of players, e.g.,

$$
\frac{\underline{\delta}_{i} \lambda_{i}^{\nu}}{\beta^{\nu}} \leq \frac{\lambda_{i}^{\nu^{\prime}}}{\beta^{\nu^{\prime}}} \leq \frac{\bar{\delta}_{i} \lambda_{i}^{\nu}}{\beta^{\nu}} \quad 1 \leq \nu<\nu^{\prime} \leq N, \quad i=1, \ldots, m
$$

where $\underline{\delta}_{i}$ and $\bar{\delta}_{i}$ are given positive numbers such that $\underline{\delta}_{i} \leq 1 \leq \bar{\delta}_{i}$ for $i=1, \ldots, m$. More generally, we may require the conditions

$$
\frac{\underline{\delta}_{i} \lambda_{i}^{\nu}}{\beta_{i}^{\nu}} \leq \frac{\lambda_{i}^{\nu^{\prime}}}{\beta_{i}^{\nu^{\prime}}} \leq \frac{\bar{\delta}_{i} \lambda_{i}^{\nu}}{\beta_{i}^{\nu}} \quad 1 \leq \nu<\nu^{\prime} \leq N, \quad i=1, \ldots, m
$$

where $\beta_{i}=\left(\beta_{i}^{\nu}\right) \in \Re_{++}^{N}, i=1, \ldots, m$ are given vectors.

These conditions can readily be included in the representation of the cone $\Lambda$ to specify restricted GNEs. Although a restricted GNE may not be unique in general, we may expect that a restricted GNE provides useful insights into outcomes of the GNEP, since it is endowed with more specific features than mere GNEs.

Another important question is about the existence of restricted GNEs. As mentioned earlier in this section, a restricted GNE exists in the special case where $\Lambda$ is given by the cone $\Lambda_{\beta}^{m}$ with $\beta \in \Re_{++}^{N}$. Therefore, more generally, the set of $\Lambda$-restricted GNEs is nonempty as long as $\Lambda \supseteq \Lambda_{\beta}^{m}$ for some $\beta \in \Re_{++}^{N}$.

\section{Computing a Restricted GNE}

In this section, we consider the GNEP with shared constraints, i.e., the GNEP in which each player $\nu$ solves $\bar{P}_{\nu}\left(x^{-\nu}\right)$, and present a heuristic method for computing a $\Lambda$-restricted GNE by solving a sequence of NEPs obtained through penalized reformulation of the GNEP. The basic idea underlying the method is to adjust the penalty parameters $\rho=\left(\rho_{i}\right)$ so that not only the feasibility is achieved for each player's problem but also the Lagrange multipliers $\lambda=\left(\lambda_{i}^{\nu}\right)$ associated with the shared constraints are led to satisfy the condition $\lambda \in \Lambda$.

In the following, we assume that the cone $\Lambda \subseteq \Re_{+}^{m N}$ is given by

$$
\Lambda=\prod_{i=1}^{m} \Lambda_{i}
$$

where $\Lambda_{i}$ is a nonempty cone such that $\Lambda_{i} \backslash\{0\} \subseteq \Re_{++}^{N}$ for each $i=1, \ldots, m$. 
For each $\nu=1, \ldots, N$, the penalty problem related to player $\nu$ 's problem $\bar{P}_{\nu}\left(x^{-\nu}\right)$ can be written as

$$
\begin{aligned}
\bar{Q}_{\nu}\left(x^{-\nu} ; \rho^{\nu}\right): & \text { minimize } \\
& f^{\nu}\left(x^{\nu}, x^{-\nu}\right)+\sum_{i=1}^{m} \rho_{i}^{\nu} g_{i}\left(x^{\nu}, x^{-\nu}\right)_{+} \\
& \text {subject to } \quad h_{j}^{\nu}\left(x^{\nu}\right) \leq 0, \quad j=1, \ldots, l_{\nu} .
\end{aligned}
$$

The game in which each player $\nu$ solves $\bar{Q}_{\nu}\left(x^{-\nu} ; \rho^{\nu}\right)$ is a NEP, which will be referred to as $\overline{\operatorname{NEP}}(\rho)$. The KKT conditions for $\bar{Q}_{\nu}\left(x^{-\nu} ; \rho^{\nu}\right)$ are given by

$$
\begin{aligned}
& \nabla_{x^{\nu}} f^{\nu}\left(x^{\nu}, x^{-\nu}\right)+\sum_{i=1}^{m} \lambda_{i}^{\nu} \nabla_{x^{\nu}} g_{i}\left(x^{\nu}, x^{-\nu}\right)+\sum_{j=1}^{l_{\nu}} \mu_{j}^{\nu} \nabla h_{j}^{\nu}\left(x^{\nu}\right)=0, \\
& 0 \leq \lambda_{i}^{\nu} \leq \rho_{i}^{\nu}, \quad i=1, \ldots, m, \\
& \xi_{i}^{\nu} \geq g_{i}\left(x^{\nu}, x^{-\nu}\right), \quad \xi_{i}^{\nu} \geq 0, \quad i=1, \ldots, m \\
& \lambda_{i}^{\nu}>0 \Rightarrow \xi_{i}^{\nu}=g_{i}\left(x^{\nu}, x^{-\nu}\right), \quad i=1, \ldots, m \\
& \lambda_{i}^{\nu}<\rho_{i}^{\nu} \Rightarrow \xi_{i}^{\nu}=0, \quad i=1, \ldots, m, \\
& h_{j}^{\nu}\left(x^{\nu}\right) \leq 0, \quad \mu_{j}^{\nu} \geq 0, \quad \mu_{j}^{\nu} h_{j}^{\nu}\left(x^{\nu}\right)=0, \quad j=1, \ldots, l_{\nu} .
\end{aligned}
$$

Note that, at a solution $x=\left(x^{1}, \ldots, x^{N}\right)$ of $\overline{\mathrm{NEP}}(\rho)$, we must have $\xi_{i}^{\nu}=\max \left\{0, g_{i}(x)\right\}$, $\nu=1, \ldots, N$ for each $i=1, \ldots, m$, and hence we have $\xi_{i}^{1}=\cdots=\xi_{i}^{N}$ for all $i=$ $1, \ldots, m$. Moreover, the following relations hold for each $i=1, \ldots, m$ :

$$
\begin{aligned}
& g_{i}(x)<0 \Rightarrow \xi_{i}^{\nu}=0, \lambda_{i}^{\nu}=0, \nu=1, \ldots, N \\
& g_{i}(x)>0 \Rightarrow \xi_{i}^{\nu}>0, \lambda_{i}^{\nu}=\rho_{i}^{\nu}, \nu=1, \ldots, N \\
& g_{i}(x)=0 \Rightarrow \xi_{i}^{\nu}=0,0 \leq \lambda_{i}^{\nu} \leq \rho_{i}^{\nu}, \nu=1, \ldots, N .
\end{aligned}
$$

The relations (9) indicate that if the $i$ th shared constraint $g_{i}(x) \leq 0$ is violated at a solution of $\overline{\operatorname{NEP}}(\rho)$, then the Lagrange multipliers $\lambda_{i}^{\nu}$ and the penalty parameters $\rho_{i}^{\nu}$ are equal in all players' problems.

This observation suggests that a $\Lambda$-restricted GNE might be obtained by solving a sequence of $\overline{\operatorname{NEP}}(\rho)$ 's, where an initial $\rho$ is sufficiently small and belongs to $\Lambda$, and subsequently, those components of $\rho$ which correspond to the violated shared constraints at a computed NE are increased in such a way that the condition $\rho \in \Lambda$ is maintained. By the convergence theorem for the Incremental Penalty Algorithm (Theorem 4.1), all the shared constraints are satisfied eventually, and a GNE is obtained. Then we check whether the Lagrange multipliers $\lambda_{i}=\left(\lambda_{i}^{\nu}\right)$ associated with the shared constraints belong to the cones $\Lambda_{i}, i=1, \ldots, m$. If any of $\lambda_{i}$ 's fails to belong to $\Lambda_{i}$, then we decrease the corresponding penalty parameters $\rho_{i}=\left(\rho_{i}^{\nu}\right)$ and resume the Incremental Penalty Algorithm. 


\section{Controlled Penalty Algorithm}

Step 0. Choose the initial penalty parameter $\rho^{0}=\left(\rho_{i}^{0}\right) \in \Lambda \backslash\{0\}$, where $\rho_{i}^{0}=\left(\rho_{i}^{0, \nu}\right) \in$ $\Re^{N}$. Set $k:=0$.

Step 1. Find a NE $x^{k}=\left(x^{k, 1}, \ldots, x^{k, N}\right)$ of $\overline{\operatorname{NEP}}\left(\rho^{k}\right)$. Let $\lambda^{k}=\left(\lambda_{i}^{k, \nu}\right), \mu^{k}=\left(\mu_{j}^{k, \nu}\right)$ and $\xi^{k}=\left(\xi_{i}^{k, \nu}\right)$ be vectors of Lagrange multipliers and artificial variables that along with $x^{k}$ satisfy the KKT conditions (8) for all $\nu=1, \ldots, N$. (Note that $\xi_{i}^{k, \nu}=\bar{\xi}_{i}^{k}:=\max \left\{0, g_{i}\left(x^{k}\right)\right\}, \nu=1, \ldots, N$ for each $i=1, \ldots, m$, as mentioned above.)

Step 2. If $\bar{\xi}_{i}^{k}=0$ for all $i=1, \ldots, m$, then a GNE is obtained; go to Step 4. Otherwise, go to Step 3.

Step 3. For each $i=1, \ldots, m$, let

$$
\rho_{i}^{k+1}:= \begin{cases}\rho_{i}^{k}+\Delta_{i}^{k} & \text { if } \bar{\xi}_{i}^{k}>0 \\ \rho_{i}^{k} & \text { if } \bar{\xi}_{i}^{k}=0\end{cases}
$$

where $\Delta_{i}^{k}=\left(\Delta_{i}^{k, \nu}\right) \in \Re_{++}^{N}$ is chosen so that $\rho_{i}^{k}+\Delta_{i}^{k} \in \Lambda_{i}$. Let $k:=k+1$ and go to Step 1.

Step 4. If $\lambda_{i}^{k} \in \Lambda_{i}$ for all $i=1, \ldots, m$, a $\Lambda$-restricted GNE is obtained; terminate. Otherwise, for each $i=1, \ldots, m$, let

$$
\rho_{i}^{k+1}:= \begin{cases}\rho_{i}^{k} & \text { if } \lambda_{i}^{k}=\rho_{i}^{k}, \\ \rho_{i}^{k}-\Delta_{i}^{k} & \text { if } \lambda_{i}^{k} \neq \rho_{i}^{k},\end{cases}
$$

where $\Delta_{i}^{k}=\left(\Delta_{i}^{k, \nu}\right) \in \Re_{++}^{N}$ is chosen so that $\rho_{i}^{k}-\Delta_{i}^{k} \in \Lambda_{i}$. (Note that $\lambda_{i}^{k} \neq \rho_{i}^{k}$ means $\lambda_{i}^{k, \nu}<\rho_{i}^{k, \nu}$ for some $\nu$.) Let $k:=k+1$ and go to Step 1 .

If $\rho_{i}^{0}, i=1, \ldots, m$ are chosen sufficiently small, we may anticipate that, at a solution of $\overline{\mathrm{NEP}}\left(\rho^{0}\right), \bar{\xi}_{i}^{0}>0$ for all shared constraints that are violated at a GNE. From the update rule of the penalty parameters, we have $\rho_{i}^{k}=\left(\rho_{i}^{k, \nu}\right) \in \Lambda_{i}, i=1, \ldots, m$ at every iteration $k$. By (9), the Lagrange multipliers $\lambda_{i}^{k}$ are equal to the penalty parameters $\rho_{i}^{k}$ for the violated shared constraints, i.e., for all $i$ such that $\bar{\xi}_{i}^{k}>0$. Thus Step 3 of the algorithm tries to guide the Lagrange multipliers $\lambda_{i}^{k}$ toward $\Lambda_{i}$ by increasing the penalty parameter $\rho_{i}^{k}$. Eventually the shared constraints will be all satisfied, i.e., $\bar{\xi}_{i}^{k}=0$ for all $i=1, \ldots, m$, and the solution at hand is a GNE (cf. Theorem 4.1). If 
the Lagrange multipliers $\lambda^{k}$ actually belong to $\Lambda$, then we are done. The GNE is in fact a $\Lambda$-restricted GNE. Otherwise, there exists at least one shared constraint such that $\lambda_{i}^{k} \neq \rho_{i}^{k}$. Then we reduce the corresponding penalty parameter $\rho_{i}$ and repeat the incremental penalty procedure.

The above procedure may be interpreted as follows: We try to compel the players to meet the shared constraints by applying Pigovian taxes, so that the resulting Nash equilibrium turns out to be a GNE in the original game. Moreover, by adaptively adjusting tax rates (penalty parameters) not only for each constraint but also for each player individually, a sequence of Nash equilibria is generated, which may be expected to converge to a restricted GNE specified by the cone $\Lambda$.

\section{$7 \quad$ Numerical Results}

We have implemented the Controlled Penalty Algorithm for finding a restricted GNE. In Step 1 of the algorithm, a Nash equilibrium $x^{k}$ of $\overline{\operatorname{NEP}}\left(\rho^{k}\right)$ is computed by applying a generalized Newton method with the Fischer-Burmeister function [11, Subsection 9.1.1] to the complementarity problem that represents the $\operatorname{KKT}$ conditions for $\overline{\operatorname{NEP}}\left(\rho^{k}\right)$.

In our numerical experiments, we used the river basin pollution game [22, Subsection 5.3.1]. In this game, there are three players and the problem of player $\nu \in\{1,2,3\}$ is given by

$$
\begin{aligned}
P_{\nu}\left(x^{-\nu}\right): \quad \text { minimize } & \left(c_{\nu} x^{\nu}+0.01\left(x^{1}+x^{2}+x^{3}\right)-\chi_{\nu}\right) x^{\nu} \\
\text { subject to } \quad x^{\nu} \geq 0 & \\
& 3.25 x^{1}+1.25 x^{2}+4.125 x^{3} \leq 100 \\
& 2.2915 x^{1}+1.5625 x^{2}+2.8125 x^{3} \leq 100
\end{aligned}
$$

with $c_{1}=0.01, c_{2}=0.05, c_{3}=0.01, \chi_{1}=2.9, \chi_{2}=2.88, \chi_{3}=2.85$. This GNEP has two shared constraints

$$
\begin{aligned}
& g_{1}(x):=3.25 x^{1}+1.25 x^{2}+4.125 x^{3} \leq 100 \\
& g_{2}(x):=2.2915 x^{1}+1.5625 x^{2}+2.8125 x^{3} \leq 100
\end{aligned}
$$

Each player $\nu$ has Lagrange multipliers $\lambda^{\nu}:=\left(\lambda_{1}^{\nu}, \lambda_{2}^{\nu}\right)^{T} \in \Re^{2}$ associated with these shared constraints. The subproblem solved in Step 1 at every iteration can be repre- 
sented as a linear complementarity problem with 15 variables

$$
\left(x^{1}, x^{2}, x^{3}, \xi_{1}^{1}, \xi_{2}^{1}, \xi_{1}^{2}, \xi_{2}^{2}, \xi_{1}^{3}, \xi_{2}^{3}, \lambda_{1}^{1}, \lambda_{2}^{1}, \lambda_{1}^{2}, \lambda_{2}^{2}, \lambda_{1}^{3}, \lambda_{2}^{3}\right)
$$

First, we let the closed convex cone $\Lambda_{\beta}^{2}$ be defined by

$$
\begin{aligned}
\Lambda_{\beta}^{2}:= & \left\{\left(\lambda_{1}^{1}, \lambda_{1}^{2}, \lambda_{1}^{3}\right)^{T} \in \Re^{3} \mid \frac{\lambda_{1}^{\nu}}{\beta^{\nu}}=\frac{\lambda_{1}^{\nu^{\prime}}}{\beta^{\nu^{\prime}}}, 1 \leq \nu \leq \nu^{\prime} \leq 3\right\} \\
& \times\left\{\left(\lambda_{2}^{1}, \lambda_{2}^{2}, \lambda_{2}^{3}\right)^{T} \in \Re^{3} \mid \frac{\lambda_{2}^{\nu}}{\beta^{\nu}}=\frac{\lambda_{2}^{\nu^{\prime}}}{\beta^{\nu^{\prime}}}, 1 \leq \nu \leq \nu^{\prime} \leq 3\right\}
\end{aligned}
$$

with $\beta:=\left(\beta^{1}, \beta^{2}, \beta^{3}\right)^{T}=(3,4,5)^{T} \in \Re^{3}$. With this choice of $\Lambda_{\beta}^{2} \subseteq \Re^{6}$, the $\Lambda_{\beta^{-}}^{2}$ restricted GNE is equivalent to the normalized equilibrium with $\beta=(3,4,5)^{T}$ (see (5)). In the algorithm, we set the initial penalty parameter $\rho^{0}=\left(\rho_{1}^{0}, \rho_{2}^{0}\right) \in \Re^{6}$ to be $\rho_{1}^{0}=(0.3,0.4,0.5)^{T}$ and $\rho_{2}^{0}=(0.15,0.2,0.25)^{T}$. In Step 3 of the $k$ th iteration, we update the penalty parameters as

$$
\rho_{i}^{k+1}:=\left\{\begin{array}{ll}
(1+0.2 / k) \rho_{i}^{k} & \text { if } \bar{\xi}_{i}^{k}>10^{-6} \\
\rho_{i}^{k} & \text { otherwise, }
\end{array} \quad i=1,2 .\right.
$$

In Step 4, we judge that the obtained GNE $x^{k}$ is a $\Lambda_{\beta}^{2}$-restricted GNE if

$$
\left|\frac{\lambda_{i}^{\nu}}{\beta^{\nu}}-\frac{\lambda_{i}^{\nu^{\prime}}}{\beta^{\nu^{\prime}}}\right|<10^{6} \quad i=1,2, \nu, \nu^{\prime}=1,2,3 .
$$

If these conditions are not met, the penalty parameters are updated as

$$
\rho_{i}^{k+1}:=\left\{\begin{array}{ll}
\rho_{i}^{k} & \text { if }\left|\lambda_{i}^{k}-\rho_{i}^{k}\right|<10^{-6} \\
\rho_{i}^{k} /(1+0.2 / k) & \text { otherwise, }
\end{array} i=1,2 .\right.
$$

Notice that the condition $\rho^{k} \in \Lambda_{\beta}^{2}$ is maintained for all $k$. In the course of computation, the algorithm obtained many GNEs that were not a restricted GNE, and finally it successfully terminated after 151 iterations by finding a $\Lambda_{\beta}^{2}$-restricted GNE, i.e., a normalized equilibrium

$$
\left(x^{1}, x^{2}, x^{3}\right)^{T}=(25.2181,14.4329,0.0000)^{T}
$$

and Lagrange multipliers of the three players

$$
\lambda^{1}=(0.5375,0.0000)^{T}, \lambda^{2}=(0.7167,0.0000)^{T}, \lambda^{3}=(0.8959,0.0000)^{T} .
$$

Note that the second shared constraint is inactive at the computed solution. 
Next, we incorporate the condition (7) and define the closed convex cone $\Lambda \subseteq \Re^{6}$ by

$$
\begin{aligned}
\Lambda:= & \left\{\left(\lambda_{1}^{1}, \lambda_{1}^{2}, \lambda_{1}^{3}\right)^{T} \in \Re^{3} \mid \frac{\delta_{1} \lambda_{1}^{\nu}}{\beta^{\nu}} \leq \frac{\lambda_{1}^{\nu^{\prime}}}{\beta^{\nu^{\prime}}} \leq \frac{\bar{\delta}_{1} \lambda_{1}^{\nu}}{\beta^{\nu}}, 1 \leq \nu \leq \nu^{\prime} \leq 3\right\} \\
& \times\left\{\left(\lambda_{2}^{1}, \lambda_{2}^{2}, \lambda_{2}^{3}\right)^{T} \in \Re^{3} \mid \frac{\delta_{2} \lambda_{2}^{\nu}}{\beta^{\nu}} \leq \frac{\lambda_{2}^{\nu^{\prime}}}{\beta^{\nu^{\prime}}} \leq \frac{\bar{\delta}_{2} \lambda_{2}^{\nu}}{\beta^{\nu}}, 1 \leq \nu \leq \nu^{\prime} \leq 3\right\}
\end{aligned}
$$

with $\beta:=\left(\beta^{1}, \beta^{2}, \beta^{3}\right)^{T}=(3,4,5)^{T} \in \Re^{3},\left(\underline{\delta}_{1}, \underline{\delta}_{2}\right)^{T}=(0.8,0.75)^{T}$ and $\left(\bar{\delta}_{1}, \bar{\delta}_{2}\right)^{T}=$ $\left(1 / \underline{\delta}_{1}, 1 / \underline{\delta}_{2}\right)^{T}$. We chose the same initial values of the penalty parameters as above, i.e., $\rho_{1}^{0}=(0.3,0.4,0.5)^{T}$ and $\rho_{2}^{0}=(0.15,0.2,0.25)^{T}$. Also we used the same penalty update rule as above, but the conditions to check a restricted GNE were modified as

$$
\frac{\underline{\delta}_{i} \lambda_{i}^{\nu}}{\beta^{\nu}} \leq \frac{\lambda_{i}^{\nu^{\prime}}}{\beta^{\nu^{\prime}}} \leq \frac{\bar{\delta}_{i} \lambda_{i}^{\nu}}{\beta^{\nu}}, \quad 1 \leq \nu<\nu^{\prime} \leq 3, \quad i=1,2
$$

The algorithm successfully obtained a restricted GNE at the 13th iteration. The restricted GNE, which was actually the first GNE obtained in the course of computation, and the corresponding Lagrange multipliers are

$$
\begin{aligned}
\left(x^{1}, x^{2}, x^{3}\right)^{T} & =(25.0622,14.8383,0.0000)^{T}, \\
\lambda^{1}=(0.5382,0.0000)^{T}, \lambda^{2} & =(0.6790,0.0000)^{T}, \lambda^{3}=(0.7276,0.0000)^{T},
\end{aligned}
$$

which slightly differ from the normalized equilibrium and the corresponding Lagrange multipliers obtained in the previous experiment. Note that the second shared constraint is also inactive at the computed solution.

Next we solved the same problem except that $\Lambda$ is given by $(11)$ with $\beta=(4,3,2)^{T}$. The initial values of the penalty parameters were set to be $\rho_{1}^{0}=(0.4,0.3,0.2)^{T}$ and $\rho_{2}^{0}=(0.4,0.3,0.2)^{T}$. With the same penalty update rules and stopping conditions, the algorithm found the following restricted GNE and Lagrange multipliers in 72 iterations:

$$
\begin{aligned}
\left(x^{1}, x^{2}, x^{3}\right)^{T} & =(0.0000,14.3915,19.8814)^{T}, \\
\lambda^{1}=(0.9951,0.0000)^{T}, \lambda^{2} & =(0.7634,0.0000)^{T}, \lambda^{3}=(0.5114,0.0000)^{T} .
\end{aligned}
$$

When we set $\Lambda$ as in (11) with $\beta=(5,3,4)^{T}$ and the initial values of the penalty parameters were chosen as $\rho_{1}^{0}=(0.5,0.3,0.4)^{T}$ and $\rho_{2}^{0}=(0.25,0.15,0.2)^{T}$, the algorithm was able to find the following restricted GNE and Lagrange multipliers only in 4 iterations:

$$
\begin{aligned}
\left(x^{1}, x^{2}, x^{3}\right)^{T} & =(14.6711,17.7709,7.2982)^{T} \\
\lambda^{1}=(0.6346,0.0000)^{T}, \lambda^{2} & =(0.4222,0.0000)^{T}, \lambda^{3}=(0.5592,0.0000)^{T} .
\end{aligned}
$$


We have also tested different choices of $\Lambda$ and the algorithm was able to find a restricted GNE in many cases. In particular, the second shared constraint was always inactive at the computed restricted GNEs. This is consistent with the observation made in [23], where the authors tried to find as many GNEs as possible and observed that the first shared constraint was always active at computed GNEs, but the second was not.

The above numerical results with the controlled penalty algorithm are summarized as follows. First we computed a (special) restricted GNE with $\Lambda_{\beta}^{2}$ given by (10), which is nothing but Rosen's normalized equilibrium with weights $\beta=\left(\beta^{\nu}\right)$, and confirmed that the algorithm successfully found it. Then we tried to compute more general restricted GNEs where $\Lambda$ is given by (11) with different values of $\beta$. Since those restricted GNEs constitute a set of GNEs that contains a normalized equilibrium, finding one of those restricted GNEs generally requires less computational effort than finding a normalized equilibrium. However, it should also be mentioned that, for some choices of $\Lambda$, the algorithm sometimes encountered a difficulty in finding a restricted GNE, although many GNEs were found in the course of computation. Moreover, in other cases, the subproblem $\overline{\operatorname{NEP}}\left(\rho^{k}\right)$, which was reformulated as a linear complementarity problem, could not be solved at some iterations, possibly due to the lack of monotonicity. It is not clear whether those failures are attributed to a defect of the algorithm or the lack of desirable properties in the problem (or subproblems) formulated with the chosen $\Lambda$.

\section{Conclusion}

Since there are usually too many GNEs, we have proposed a new solution concept called a restricted GNE, which can be characterized by certain properties enjoyed by the players' Lagrange multipliers (shadow prices) associated with the shared constraints. This concept contains as a special case the classical solution concept called a normalized equilibrium. We have also proposed two algorithms; the incremental penalty algorithm and the controlled penalty algorithm. The first algorithm is designed to find a GNE and its convergence theorem is established. The second algorithm, which includes the first algorithm as a subprocedure, is designed to find a restricted GNE and its computational procedure can be regarded as a Pigovian taxation process. The second algorithm is a heuristic method. Through numerical experiments on a simple GNEP, we have shown that the algorithm can find a restricted GNE for various conditions imposed on the Lagrange multipliers. However, the algorithm sometimes failed to obtain a restricted 
GNE, which may be due to the nonexistence of a restricted GNE. As mentioned at the end of Section 5 , there exits a $\Lambda$-restricted GNE when $\Lambda$ contains the cone $\Lambda_{\beta}^{m}$ for some $\beta \in \Re^{++}$. It is a future subject to study existence conditions for restricted GNEs under a more general choice of the cone $\Lambda$.

Acknowledgements. The author is grateful to the referees for their helpful comments.

\section{References}

[1] K.J. Arrow and G. Debreu, Existence of an equilibrium for a competitive economy, Econometrica 22 (1954), pp. 265-290.

[2] J.P. Aubin, Mathematical Methods of Game and Economic Theory, North-Holland, Amsterdam, 1979.

[3] J.M. Borwein and A.S. Lewis, Convex Analysis and Nonlinear Optimization: Theory and Examples, Second Edition, Springer, 2006.

[4] M. Breton, G. Zaccour and M. Zahaf, A game-theoretic formulation of joint implementation of environmental projects, European Journal of Operational Research 168 (2006), pp. 221-239.

[5] J. Contreras, M. Klusch and J.B. Krawczyk, Numerical solutions to Nash-Cournot equilibria in coupled constraint electricity markets, IEEE Transactions on Power Systems 19 (2004), pp. 195-206.

[6] G. Debreu, A social equilibrium existence theorem, Proceedings of the National Academy of Sciences 38 (1952), pp. 886-893.

[7] G. Di Pillo and L. Grippo, Exact penalty functions in constrained optimization, SIAM Journal on Control and Optimization 27 (1989), pp. 1333-1360.

[8] F. Facchinei, A. Fischer and V. Piccialli, On generalized Nash games and variational inequalities, Operations Research Letters 35 (2007), pp. 159-164.

[9] F. Facchinei, A. Fischer and V. Piccialli, Generalized Nash equilibrium problems and Newton methods, Mathematical Programming, 117 (2009), pp. 163-194. 
[10] F. Facchinei and C. Kanzow, Generalized Nash equilibrium problems, $4 O R 5$ (2007), pp. 173-210.

[11] F. Facchinei and J.-S. Pang, Finite-Dimensional Variational Inequalities and Complementarity Problems, Volume 2, Springer-Verlag, New York, 2003.

[12] F. Facchinei and J.-S. Pang, Exact penalty functions for generalized Nash problems, G. Di Pillo and M. Roma (eds.), Large-Scale Nonlinear Optimization, Springer-Verlag, Heidelberg, 2006, pp. 115-126.

[13] M. Fukushima, A class of gap functions for quasi-variational inequality problems, Journal of Industrial and Management Optimization 3 (2007), pp. 165-171.

[14] S.-P. Han and O.L. Mangasarian, Exact penalty functions in nonlinear programming, Mathematical Programming 17 (1979), pp. 251-269.

[15] P.T. Harker, Generalized Nash games and quasi-variational inequalities, European Journal of Operational Research 54 (1991), pp. 81-94.

[16] A. Haurie and J.B. Krawczyk, Optimal charges on river effluent from lumped and distributed sources, Environmental Modeling and Assessment 2 (1997), pp. 177189.

[17] A. von Heusinger and C. Kanzow, Optimization reformulations of the generalized Nash equilibrium problem using Nikaido-Isoda-type functions. Computational $O p$ timization and Applications, DOI 10.1007/s10589-007-9145-6.

[18] B.F. Hobbs and J.-S. Pang, Nash-Cournot equilibrium in electric power markets with piecewise linear demand functions and joint constraints, Operations Research 55 (2007), pp. 113-127.

[19] A. Kesselman, S. Leonardi and V. Bonifaci, Game-theoretic analysis of internet switching with selfish users, Proceedings of the First International Workshop on Internet and Network Economics, WINE, Lecture Notes in Computer Science 3828 (2005), pp. 236-245.

[20] J.B. Krawczyk, Coupled constraint Nash equilibria in environmental games, Resource and Energy Economics 27 (2005), pp. 157-181.

[21] J.B. Krawczyk, Numerical solutions to coupled-constraint (or generalised Nash) equilibrium problems, Computational Management Science 4 (2007), pp. 183-204. 
[22] J.B. Krawczyk and S. Uryasev, Relaxation algorithms to find Nash equilibria with economic applications, Environmental Modeling and Assessment 5 (2000), pp. 6373.

[23] K. Nabetani, P. Tseng and M. Fukushima, Parametrized variational inequality approaches to generalized Nash equilibrium problems with shared constraints, Technical Report 2008-011, Department of Applied Mathematics and Physics, Kyoto University, October 2008.

[24] H. Nikaido and K. Isoda, Note on noncooperative convex games, Pacific Journal of Mathematics 5 (1955), pp. 807-815.

[25] J.-S. Pang and M. Fukushima, Quasi-variational inequalities, generalized Nash equilibria, and multi-leader-follower games, Computational Management Science 2 (2005), pp. 21-56.

[26] J.-S. Pang, G. Scutari, F. Facchinei and C. Wang, Distributed power allocation with rate constraints in Gaussian parallel interference channels, IEEE Transactions on Information Theory 54 (2008), pp. 3471-3489.

[27] S.M. Robinson, Shadow prices for measures of effectiveness, I: Linear model, $O p$ erations Research 41 (1993), pp. 518-535.

[28] S.M. Robinson, Shadow prices for measures of effectiveness, II: General model, Operations Research 41 (1993), pp. 536-548.

[29] J.B. Rosen, Existence and uniqueness of equilibrium points for concave $N$-person games, Econometrica 33 (1965), pp. 520-534.

[30] J.-Y. Wei and Y. Smeers, Spatial oligopolistic electricity models with Cournot generators and regulated transmission prices, Operations Research 47 (1999), pp. 102112. 